\title{
THREE CARDINAL FUNCTIONS SIMILAR TO NET WEIGHT
}

\author{
ROY A. JOHNSON, ELIZA WAJCH, AND WLADYSLAW WILCZYŃSKI
}

(Communicated by Dennis Burke)

\begin{abstract}
The purpose of this paper is to introduce and investigate cardinal functions called pseudonet weight, weak net weight, and weak pseudonet weight. These are similar to but generally smaller than net weight. We look at how these cardinal functions relate to hereditary Lindelöf degree, hereditary density, and spread, and we study their behavior under products.
\end{abstract}

An important and useful cardinal function for a topological space is that of weight, namely, the minimum cardinal for a base of open sets. Net weight is similar to weight, except that "base" members need not be open. In this paper we look at three cardinal functions which are slight variations of net weight.

Throughout this paper, $\kappa$ denotes an infinite cardinal number, and for simplicity, all cardinal functions will be infinite. The smallest (infinite) cardinal number $\kappa$ such that $X$ is hereditarily $\kappa$-Lindelöf (hereditarily $\kappa$-separable, resp.) is denoted by $h l(X)$ ( $h d(X)$, resp.). The spread of $X$ (equivalently, hereditary Souslin number) is denoted by $s(X)$. As usual, $w(X)$ denotes the weight of $X$. The set of all real numbers is denoted by $R$. For notation and terminology not defined here, see [1].

In $\S 1$ we generalize the notion of nets (also called networks) by introducing $\kappa$-pseudonets. Some examples are given of (nonregular) Hausdorff spaces which have $\kappa$-pseudonets but have no nets of cardinality $\leq \kappa$. Theorem 1.9 shows that pseudonet weight coincides with net weight in regular spaces. We also examine weak net weight and weak pseudonet weight. In terms of definition, weak net weight is to net weight as weak pseudonet weight is to pseudonet weight.

The main theorem in $\S 2$ is Theorem 2.3, which shows that the Cartesian product $X \times Y$ of a hereditarily $\kappa$-Lindelöf space $X$ and a space $Y$ having a $\kappa$-pseudonet is hereditarily $\kappa$-Lindelöf.

\section{DEFINITIONS, EXAMPLES, AND ELEMENTARY RELATIONSHIPS}

1.1. Definition (cf. [1, Remark 3.1.17, p. 170]). A family $\mathscr{E}$ of subsets of a topological space $X$ is called a net in $X$ if and only if for each open set

Received by the editors March 2, 1989 and, in revised form, July 21, 1989.

1980 Mathematics Subject Classification (1985 Revision). Primary 54A25.

Key words and phrases. Net weight, pseudonet weight, weak net weight, weak pseudonet weight, hereditary Lindelöf degree, hereditary density, spread. 
$U \subset X$ and $x \in U$, there exists $E \in \mathscr{E}$ such that $x \in E$ and $E \subset U$. It will be convenient to say that a family $\mathscr{E}$ of subsets of a topological space $X$ is a $\kappa$-net in $X$ if and only if $|\mathscr{E}| \leq \kappa$ and $\mathscr{E}$ is a net. The cardinal number

$$
n w(X)=\min \{\kappa \geq \omega \text { : there exists a } \kappa \text {-net in } X\}
$$

is called the net weight of $X$.

1.2. Definition. A family $\mathscr{E}$ of subsets of a topological space $X$ will be called a $\kappa$-pseudonet in $X$ if and only if $|\mathscr{E}| \leq \kappa$ and for each open set $U \subset X$ and $x \in U$, there exists $E \in \mathscr{E}$ such that $x \in E$ and $|E \backslash U| \leq \kappa$. The cardinal number

$$
p n(X)=\min \{\kappa \geq \omega \text { : there exists a } \kappa \text {-pseudonet in } X\}
$$

will be called the pseudonet weight of $X$.

1.3. Definition. A family $\mathscr{E}$ of subsets of a space $X$ will be called a weak $\kappa$-net in $X$ if and only if $|\mathscr{E}| \leq \kappa$; and for each open set $U \subset X$, there exists a set $A \subset U$ such that $|A| \leq \kappa$; and for each $x \in U \backslash A$, there exists $E \in \mathscr{E}$ such that $x \in E \subset U$. The cardinal number

$$
w n w(X)=\min \{\kappa \geq \omega: \text { there exists a weak } \kappa \text {-net in } X\}
$$

will be called the weak net weight of $X$.

1.4. Definition. A family $\mathscr{E}$ of subsets of a space $X$ will be called a weak $\kappa$-pseudonet in $X$ if and only if $|\mathscr{E}| \leq \kappa$; and for each open set $U \subset X$, there exists a set $A \subset U$ such that $|A| \leq \kappa$; and for each $x \in U \backslash A$, there exists $E \in \mathscr{E}$ such that $x \in E$ and $|E \backslash U| \leq \kappa$. The cardinal number

$$
w p n(X)=\min \{\kappa \geq \omega \text { : there exists a weak } \kappa \text {-pseudonet in } X\}
$$

will be called the weak pseudonet weight of $X$.

So to speak, a family $\mathscr{E}$ is a net if for each open set, each point of that open set lies in a member of $\mathscr{E}$ which is contained in the open set. $\mathscr{E}$ is a pseudonet if for each open set, each point of that open set lies in a member of $\mathscr{E}$ which is almost contained in the open set. $\mathscr{E}$ is a weak net if for each open set, almost each point of that open set lies in a member of $\mathscr{E}$ which is contained in the open set. Finally, $\mathscr{E}$ is a weak pseudonet if for each open set, almost each point of that open set lies in a member of $\mathscr{E}$ which is almost contained in the open set. In all this discussion, "almost" means that the exceptional set has cardinality at most $\kappa$.

Of course, every $\kappa$-net is both a $\kappa$-pseudonet and a weak $\kappa$-net. Hence, we have $p n(X) \leq n w(X)$ and $w n w(X) \leq n w(X)$. Similarly, every $\kappa$-pseudonet and every weak $\kappa$-net is a weak $\kappa$-pseudonet, so that $w p n(X) \leq p n(X)$ and $w p n(X) \leq w n w(X)$. In general, no other inequalities hold between these four cardinal functions. Having net weight $\leq \kappa$, pseudonet weight $\leq \kappa$, weak net weight $\leq \kappa$, or weak pseudonet weight $\leq \kappa$ are hereditary properties. More precisely, if $Y$ is a subset of $X$ and $\mathscr{E}$ is a $\kappa$-net ( $\kappa$-pseudonet, weak $\kappa$-net, weak $\kappa$-pseudonet, resp.) in $X$, then so is $\{E \cap Y: E \in \mathscr{E}\}$ in the subspace $Y$. 
1.5. Theorem. For every space $X$, the inequality $h l(X) \leq p n(X)$ holds.

Proof. It is easy to prove this statement directly, but it is worth observing that this theorem follows as an immediate corollary of Theorem 2.3.

1.6. Theorem. For every space $X$, the inequality $h d(X) \leq w n w(X)$ holds.

Proof. Since $w n w(X)$ is hereditary, it suffices to prove that $d(X) \leq w n x(X)$. Suppose that $\mathscr{E}$ is a weak $\kappa$-net in $X$. Let $A$ be a subset of $X$ formed by choosing one point from each nonempty $E \cap X$ such that $E \in \mathscr{E}$. Then $|A| \leq \kappa$.

We show that $|X \backslash \bar{A}| \leq \kappa$. Assuming otherwise that $|X \backslash \bar{A}|>\kappa$, we obtain that there exist $x \in X \backslash \bar{A}$ and $E \in \mathscr{E}$ such that $x \in E$ and $E \subset X \backslash \bar{A}$. Then $E \cap X \neq \varnothing$ so that $A \cap E \neq \varnothing$ by the choice of $A$. But this is impossible since $\bar{A} \cap E=\varnothing$. Hence, $|X \backslash \bar{A}| \leq \kappa$ and $|A \cup(X \backslash \bar{A})| \leq \kappa$ also. As $A \cup(X \backslash \bar{A})$ is dense in $X$, we have that $d(X) \leq \kappa$.

1.7. Theorem. For every space $X$, the inequality $s(X) \leq w p n(X)$ holds.

Proof. Let $w p n(X)=\kappa$ and suppose that $s(X)>\kappa$. Then there exists a discrete subspace $Y$ of $X$ such that $|Y|>\kappa$. Of course, $Y$ can be partitioned into a family of subsets $\left\{U_{s}: s \in S\right\}$ such that each $U_{s}$ has cardinality greater than $\kappa$ and such that $|S|>\kappa$. Necessarily, each $U_{s}$ is open in $Y$. Because weak pseudonet weight is a hereditary property, we may suppose that $\left\{E_{t}: t \in T\right\}$ is a weak pseudonet for $X$ such that $|T|=\kappa$. If $s \in S$ and $t \in T$, let us say that $E_{t}$ helps $U_{s}$ if and only if $\left|U_{s} \cap E_{t}\right|>\kappa$ and $\left|E_{t} \backslash U_{s}\right| \leq \kappa$. Evidently, no $E_{t}$ can help more than one $U_{s}$. On the other hand, for each $s \in S$, there must exist $t \in T$ such that $E_{t}$ helps $U_{s}$. Hence, $|T| \geq|S|>\kappa$, which is impossible.

Theorem 1.7 has some immediate consequences. For example, if $X$ is a regular space, then by a theorem of Šapirovskii, $n w(X) \leq 2^{w p n(X)}$ [4, Theorem 5.3, p. 23]. The Sorgenfrey line ([1, Example 1.2.2, p. 39]) shows that this inequality cannot be sharpened. By another theorem of Śapirovskiǐ, every space $X$ has a dense subspace $Y$ with $h l(Y) \leq s(X)$, so that $h l(Y) \leq w p n(X)$ [4, Proposition 5.6, p. 24]. Since $s(X)=h l(X)$ for hereditarily paracompact spaces, we have $h l(X) \leq w p n(X)$ if $X$ is such a space.

1.8. Theorem. For every space $X$, the inequality $h d(X) \leq w p n(X)^{+}$holds, where $w p n(X)^{+}$denotes the smallest cardinal greater than wpn $(X)$.

Proof. Let $\kappa=w p n(X)$, let $\mathscr{E}$ be a weak $\kappa$-pseudonet in $X$, and let $Y \subset X$. Denote $\mathscr{E}^{*}=\{E \in \mathscr{E}:|E \cap Y|>\kappa\}$. Suppose $\mathscr{E}^{*} \neq \varnothing$. To each $E \in \mathscr{E}^{*}$ assign a set $A_{E} \subset E \cap Y$ such that $\left|A_{E}\right|=\kappa^{+}$. Put

$$
A=\bigcup\left\{E \cap Y: E \in \mathscr{E} \backslash \mathscr{E}^{*}\right\} \cup \bigcup\left\{A_{V}: E \in \mathscr{E} \backslash \mathscr{E}^{*}\right\} .
$$

We show that $|Y \backslash \bar{A}| \leq \kappa$. Assuming otherwise, there exists $E \in \mathscr{E}$ such that $E \cap(Y \backslash \bar{A}) \neq \varnothing$ and $|E \cap \bar{A}| \leq \kappa$. Obviously, $E \in \mathscr{E}^{*}$; otherwise $E \cap Y \subset A$. Since $\left|A_{E}\right|=\kappa^{+}$, we have $A_{E} \backslash \bar{A} \neq \varnothing$, which is impossible. 
To prove the next two theorems, we need the following definition. A point $x$ of a space $X$ will be called a $\kappa$-condensation point of a set $E \subset X$ if and only if for every open neighborhood $U$ of $x$, the inequality $|U \cap E|>\kappa$ holds. Denote by $E^{(\kappa)}$ the set of all $\kappa$-condensation points of $E$.

Let us observe that if $E$ is a subset of a hereditarily $\kappa$-Lindelöf space, then the set $E \backslash E^{(\kappa)}$ can be covered by a family $\mathscr{U}$ of open sets such that $|\mathscr{U}| \leq \kappa$ and $|U \cap E| \leq \kappa$ for each $U \in \mathscr{U}$; hence $\left|E \backslash E^{(\kappa)}\right| \leq \kappa$.

1.9. Theorem. If $X$ is a regular (not necessarily $T_{1}$ ) space, then $p n(X)=$ $n w(X)$.

Proof. It suffices to show that $n w(X) \leq p n(X)$. Put $\kappa=p n(X)$ and let $\mathscr{E}$ be a $\kappa$-pseudonet in $X$. Because $X$ is hereditarily $\kappa$-Lindelöf, the family

$$
\mathscr{N}=\left\{E^{(\kappa)}: E \in \mathscr{E}\right\} \cup\left\{\{x\}: x \in E \backslash E^{(\kappa)} \text { for some } E \in \mathscr{E}\right\}
$$

is of cardinality $\leq \kappa$. If $U$ is an open subset of $X$ and $x \in U$, then we can choose an open set $V$ such that $x \in V$ and $\bar{V} \subset U$, and we can choose $E \in \mathscr{E}$ such that $x \in E$ and $|E \backslash V| \leq \kappa$. As $|(X \backslash \bar{V}) \cap E| \leq \kappa$, then $E^{(\kappa)} \subset \bar{V}$, which implies that $\mathscr{N}$ is a net in $X$. Hence, $n w(X) \leq \kappa$.

1.10. Theorem. Suppose that a space $X$ is regular (not necessarily $T_{1}$ ) and hereditarily $\kappa$-Lindelöf. If $X$ has a weak $\kappa$-pseudonet, then $X$ has a weak $\kappa$-net consisting of closed sets. Hence, if $X$ is regular, then

$$
w n w(X) \leq \max \{w p n(X), h l(X)\} .
$$

Proof. Let $\mathscr{E}$ be a weak $\kappa$-pseudonet in $X$. Denote

$$
\mathscr{N}=\left\{E^{(\kappa)}: E \in \mathscr{E}\right\} \cup\left\{\overline{\{x\}}: x \in E \backslash E^{(\kappa)} \text { for some } E \in \mathscr{E}\right\} .
$$

Because $h l(X) \leq \kappa$, then $|\mathcal{N}| \leq \kappa$. It is obvious that all members of $\mathscr{N}$ are closed. We shall show that $\mathcal{N}$ is a weak $\kappa$-net.

Suppose that $U$ is an open set in $X$. There exists a collection $\left\{U_{s}: s \in S\right\}$ of open subsets of $X$ such that $|S| \leq \kappa$ and $U=\bigcup\left\{\bar{U}_{s}: s \in S\right\}=\bigcup\left\{U_{s}: s \in\right.$ $S\}$. For each $s \in S$, there exists a set $A_{s} \subset U_{s}$ such that $\left|A_{s}\right| \leq \kappa$ and if $x \in U_{s} \backslash A_{s}$, then there exists $E \in \mathscr{E}$ such that $x \in E$ and $\left|E \backslash U_{s}\right| \leq \kappa$. The set $A=\bigcup\left\{A_{s}: s \in S\right\}$ is of cardinality $\leq \kappa$. If $x \in U \backslash A$, then $x \in U_{s} \backslash A_{s}$ for some $s \in S$. Hence, there exists $E \in \mathscr{E}$ such that $x \in E$ and $\left|E \backslash U_{s}\right| \leq \kappa$. If $x \in E \backslash E^{(\kappa)}$, then $\overline{\{x\}} \in \mathcal{N}$ and $\overline{\{x\}} \subset \bar{U}_{s} \subset U$. On the other hand, if $x \in E^{(\kappa)}$, then the proof of Theorem 1.9 shows that $E^{(\kappa)} \subset \bar{U}_{s} \subset U$.

1.11. Theorem. If $X$ is regular (not necessarily $T_{1}$ ), then the inequality $h d(X) \leq w p n(X)$ holds.

Proof. By the remarks following Theorem 1.7, $X$ contains a dense subset $Y$ such that $h l(Y) \leq w p n(X)$. Then by Theorem 1.10 , wnw $(Y) \leq w p n(X)$, so that $h d(Y) \leq w p n(X)$ by Theorem 1.6. Since $Y$ is dense in $X$, it follows that $d(X) \leq w p n(X)$, so that $h d(X) \leq w p n(X)$. 
By combining Theorems 1.10 and 1.11 , we notice that $w p n(X)=w n w(X)$ if $X$ is a regular space for which $h l(X) \leq h d(X)$. Moreover, if $X$ is a metrizable space, then $h d(X)=w(X)$ so that $w p n(X)=w(X)$ in that case.

1.12. Theorem. If a space $Y$ is a continuous image of a space $X$, then $p n(Y) \leq p n(X), w n w(Y) \leq w n w(X)$, and $w p n(Y) \leq w p n(X)$.

Proof. Suppose that $\phi: X \rightarrow Y$ is a continuous surjection. If $\mathscr{E}$ is a $\kappa$ pseudonet [resp., weak $\kappa$-net, weak $\kappa$-pseudonet] in $X$, then the family $\{\phi(E)$ : $E \in \mathscr{E}\}$ is a $\kappa$-pseudonet [resp., weak $\kappa$-net, weak $\kappa$-pseudonet] in $Y$. We show, for example, that $\{\phi(E): E \in \mathscr{E}\}$ is a weak $\kappa$-pseudonet in $Y$ if $\mathscr{E}$ is a weak $\kappa$-pseudonet in $X$. Suppose $U$ is an open set in $Y$. Then there exists a set $A \subset \phi^{-1}(U)$ such that for each $x \in \phi^{-1}(U) \backslash A$, there exists $E \in \mathscr{E}$ such that $x \in E$ and $\left|E \backslash \phi^{-1}(U)\right| \leq \kappa$. Of course, $|\phi(A)| \leq \kappa$. Now suppose $y \in U \backslash \phi(A)$. Then there exists $x \in \phi^{-1}(U) \backslash A$ such that $\phi(x)=y$. Choose $E \in \mathscr{E}$ such that $x \in E$ and such that $\left|E \backslash \phi^{-1}(U)\right| \leq \kappa$. Then $y \in \phi(E)$ and $\phi(E) \backslash U \subset \phi\left[E \backslash \phi^{-1}(U)\right]$, so that $|\phi(E) \backslash U| \leq \kappa$. The proofs of the remaining two statements are entirely similar.

Now, let us look at some examples of Hausdorff spaces for which the pseudonet weight is different from the net weight. It will follow from Theorem 1.9 that neither of these spaces is regular.

1.13. Example. $\left(\left[w p w\left(X_{0}\right)=p n\left(X_{0}\right)=\omega\right.\right.$ and $\left.\left.w_{1}=w n w\left(X_{0}\right)=n w\left(X_{0}\right)\right]\right)$. (cf. [7, Example 2, p. 179].) Let $X_{0}=\left[0, \omega_{1}\right)$ and let $\phi: X_{0} \rightarrow R$ be a one-toone function. Consider the topology on $X_{0}$ generated by the base consisting of all sets of the form $\phi^{-1}(U) \cap\left[\alpha, \omega_{1}\right)$ where $\alpha \in X_{0}$ and $U$ is open in $R$ with the usual topology. Then $X_{0}$ is Hausdorff and nonseparable, so $n w\left(X_{0}\right)=\omega_{1}$. If $\mathscr{B}$ is a countable base for the usual topology on $R$, then the family $\mathscr{E}=$ $\left\{\phi^{-1}(B): B \in \mathscr{B}\right\}$ is an $\omega$-pseudonet in $X_{0}$.

1.14. Example. $\left(\left[w p w\left(X_{1}\right)=p n\left(X_{1}\right)=\omega\right.\right.$ and $\left.\left.\omega<w n w(X) \leq n w(X)\right]\right)$. Let $X_{1}=R^{2}$ be considered with the topology consisting of all sets of the form $U \backslash A$, where $U$ is an open set in $R^{2}$ with the usual topology and $A$ is a countable subset of $X_{2}=R \times\{0\}$. Then every countable base for the usual topology on $R^{2}$ is an $\omega$-pseudonet in $X_{1}$. Because $X_{2}$ is a nonseparable subspace of $X_{1}$ (cf. [1, Problem 2.7.9(f), p. 155]), then $n w\left(X_{1}\right)>\omega$. Obviously, the space $X_{1}$ is separable and Hausdorff.

Examples 1.13 and 1.14 show that $h d(X)$ can be greater than $p n(X)$ and hence greater than $\operatorname{wp} n(X)$. Thus the inequality of Theorem 1.8 cannot be improved upon. These examples also point out that the assumption of regularity cannot be omitted in Theorems 1.10 and 1.11 .

Let us look at an example similar to Example 1.13 for which weak net weight differs from net weight and from pseudonet weight.

1.15. Example. $\left(\left[w p w\left(X_{3}\right)=w n w\left(X_{3}\right)=\omega\right.\right.$ and $\left.\left.\omega_{1}=p n\left(X_{3}\right)=n w\left(X_{3}\right)\right]\right)$. (Cf. [3, Theorem 2, p. 118] or [7, Example 1, p. 179]). Denote $X_{3}=\left[0, \omega_{1}\right)$ 
and let $\phi: X_{3} \rightarrow R$ be a one-to-one function. Take a countable base $\mathscr{B}$ for the usual topology on $R$ and consider the topology on $X_{3}$ generated by the base consisting of all sets of the form $\phi^{-1}(U) \cap[0, \alpha)$, where $U \in \mathscr{B}$ and $\alpha \leq \omega_{1}$. Then $X_{3}$ is a Hausdorff space which is not Lindelöf. Hence, $X_{3}$ does not have an $\omega$-net or an $\omega$-pseudonet. We shall show that the family $\mathscr{E}=\left\{\phi^{-1}(U): U \in \mathscr{B}\right\}$ is a weak $\omega$-net in $X_{3}$.

Suppose that $V$ is an open set in $X_{3}$. Then $V=\bigcup\left\{\phi^{-1}\left(U_{s}\right) \cap\left[0, \alpha_{s}\right): s \in\right.$ $S\}$, where $U_{s} \in \mathscr{B}$ and $\alpha_{s} \leq \omega_{1}$ for each $s \in S$. Let $U_{1}, U_{2}, \ldots$ be an enumeration of the members of $\mathscr{B}$. For each positive integer $n$, denote $S_{n}=$ $\left\{s \in S: U_{s}=U_{n}\right\}$. If $S_{n} \neq \varnothing$, then let $\alpha_{n}=\sup \left\{\alpha_{s}: s \in S_{n}\right\}$. Then $\left[0, \alpha_{n}\right)=$ $\bigcup\left\{\left[0, \alpha_{s}\right): s \in S_{n}\right\}$. Also $V=\bigcup\left\{\phi^{-1}\left(U_{n}\right) \cap\left[0, \alpha_{n}\right): S_{n} \neq \varnothing\right\}$, and the set $A=\bigcup\left\{\left[0, \alpha_{n}\right): \alpha_{n}<\omega_{1}\right\}$ is countable. Of course, if $x \in V \backslash A$, then $x \in$ $\phi^{-1}\left(U_{n}\right) \subset V$ for some positive integer $n$.

It follows from Theorem $44 . \mathrm{H}$ of $[2$, p. 220] and our Theorem 1.6 thatunder the assumption of $[M A+\neg C H]$-every compact Hausdorff space having a weak $\omega$-net is hereditarily Lindelöf. On the other hand, Example 1.16 shows that under $C H$, a compact Hausdorff space can have a weak $\omega$-net without being hereditarily Lindelöf.

1.16. Example. $\left(\left[w p n\left(X_{4}\right)=w n w\left(X_{4}\right)=\omega\right.\right.$ and $\left.\left.\omega_{1}=p n\left(X_{4}\right)=n w\left(X_{4}\right)\right]\right)$. Using the Continuum Hypothesis, I. Juhász, K. Kunen, and M. E. Rudin constructed in $[5, \S 1]$ a locally compact, locally countable topology $\mathscr{T}$ on $R$, finer than the usual topology and such that if $U \in \mathscr{T}$, then there exists a subset $G$ of $U$ such that $G$ is open in the usual topology and $|U \backslash G| \leq \omega$. If $X_{4}$ is this space, then $X_{4}$ is not hereditarily Lindelöf but any countable base $\mathscr{E}$ for the usual topology on $R$ is a weak $\omega$-net in $X_{4}$. Indeed, for each open subset $U$ of $X_{4}$, choose $G \subset U$ such that $G$ is open in the usual topology and $|U \backslash G| \leq \omega$; if $x \in G \subset U$, then there exists $E \in \mathscr{E}$ such that $x \in E \subset G \subset U$.

1.17. Example. $\left(\left[w p n\left(X_{5}\right)=\omega\right.\right.$ and $\left.\left.\omega_{1}=p n\left(X_{5}\right)=n w\left(X_{5}\right)\right]\right)$. Using the combinatorial principle $\$$, A. J. Ostaszewski constructed in [6, p. 506] a locally countable, locally compact Hausdorff topology on $X_{5}=\left[0, \omega_{1}\right)$ such that each open set in $X_{5}$ is countable or cocountable. Because each uncountable closed subset of $X_{5}$ contains some interval $\left[\alpha, \omega_{1}\right)$, where $\alpha<\omega_{1}$, it follows that $X_{5}$ has no weak $\omega$-nets consisting of closed sets. However, it is easily seen that the family $\mathscr{E}=\{X\}$ is a weak $\omega$-pseudonet in $X_{5}$. Hence, the assumption of the hereditary $\kappa$-Lindelöf property is needed in Theorem 1.10. We do not know the value for $w n w\left(X_{5}\right)$.

\section{Applications to PRODUCTS}

Unlike net weight, the cardinal functions pseudonet weight, weak net weight, and weak pseudonet weight are not preserved under finite products. 
2.1. Theorem. If spaces $X$ and $Y$ are such that $|Y|>\kappa$ and wpn $(X \times Y) \leq$ $\kappa$, then $n w(X) \leq \kappa$. Hence, if $|Y|>\kappa$ and $p n(X \times Y) \leq \kappa$ or if $|Y|>\kappa$ and $w n w(X \times Y) \leq \kappa$, then $n w(X) \leq \kappa$.

Proof. Let $\mathscr{E}$ be a weak $\kappa$-pseudonet in $X \times Y$. For each $E \in \mathscr{E}$, denote $N(E)=\{x \in X:|E(x)|>\kappa\}$, where $E(x)=\{y \in Y:(x, y) \in E\}$. We show that the family $\{N(E): E \in \mathscr{E}\}$ is a net in $X$.

Let $U$ be an open set in $X$ and $x_{0} \in U$. There exists a subfamily $\mathscr{E}^{*}$ of $\mathscr{E}$ and a subset $Y_{0}$ of $Y$ with $\left|Y_{0}\right| \leq \kappa$ such that $\left\{x_{0}\right\} \times\left(Y \backslash Y_{0}\right) \subset \bigcup\left\{E: E \in \mathscr{E}^{*}\right\}$ and $|E \backslash(U \times Y)| \leq \kappa$ for each $E \in \mathscr{E}^{*}$. Because $|Y|>\kappa$ and hence $\left|Y \backslash Y_{0}\right|>$ $\kappa$, there exists $E_{0} \in \mathscr{E}^{*}$ such that $\left|E_{0}\left(x_{0}\right)\right|>\kappa$, so $x_{0} \in N\left(E_{0}\right)$. If $x \notin U$, then by virtue of the inequality $\left|E_{0} \backslash(U \times Y)\right| \leq \kappa$, we have that $\left|E_{0}(x)\right| \leq \kappa$; thus $x \notin N\left(E_{0}\right)$ and $N\left(E_{0}\right) \subset U$.

2.2. Corollary. For every space $X$, we have the equality:

$$
w p n(X \times X)=n w(X) .
$$

Hence $w n w(X \times X)=n w(X)$ and $p n(X \times X)=n w(X)$.

Proof. By virtue of Theorem 2.1 and the fact that $n w(X) \leq \max \{\omega,|X|\}$, we have $w p n(X \times X) \geq n w(X)$. The reverse inequality follows from the fact that $n w(X \times X)=n w(X)$.

Recall that hereditarily Lindelöf degree is not preserved under finite products (cf. [1, Examples 3.8.14 and 3.8.15, pp. 248-249]); neither is pseudonet weight (Theorem 2.1). However, we have the following:

2.3. Theorem. If $X$ and $Y$ are topological spaces such that $h l(X) \leq \kappa$ and $p n(Y) \leq \kappa$, then $h l(X \times Y) \leq \kappa$.

Proof. Suppose that $\left\{U_{s} \times V_{s}: s \in S\right\}$ is a collection of open rectangles in $X \times Y$. Put $W=\bigcup\left\{U_{s} \times V_{s}: s \in S\right\}$, and let the family $\left\{E_{t}: t \in T\right\}$, where $|T| \leq \kappa$, be a $\kappa$-pseudonet in $Y$. For each $t \in T$, denote $S_{t}=\left\{s \in S:\left|E_{t} \backslash V_{s}\right| \leq \kappa\right\}$. Because $h l(X) \leq \kappa$, there exists a set $S_{t}^{*} \subset S_{t}$ such that $\left|S_{t}^{*}\right| \leq \kappa$ and $\bigcup\left\{U_{s}: s \in S_{t}\right\}=$ $\bigcup\left\{U_{s}: s \in S_{t}^{*}\right\}$.

Let $G_{t}=\bigcap\left\{V_{s}: s \in S_{t}^{*}\right\}$. Then, by the definition of $S_{t}^{*}$, the set $E_{t} \backslash G_{t}=$ $\bigcup\left\{E_{t} \backslash V_{s}: s \in S_{t}^{*}\right\}$ has cardinality $\leq \kappa$; thus the set $H=\bigcup\left\{E_{t} \backslash G_{t}: t \in T\right\}$ is of cardinality $\leq \kappa$. Using the assumption that $h l(X) \leq \kappa$, we obtain that, for each $y \in H$, there exists a set $S^{*}(y) \subset\left\{s \in S: y \in V_{s}\right\}$ such that $\left|S^{*}(y)\right| \leq \kappa$ and

$$
\bigcup\left\{U_{s}: s \in S \text { and } y \in V_{s}\right\}=\bigcup\left\{U_{s}: s \in S^{*}(y)\right\} .
$$

Let $S^{*}=\bigcup\left\{S_{t}^{*}: t \in T\right\} \cup \bigcup\left\{S^{*}(y): y \in H\right\}$. Clearly, $\left|S^{*}\right| \leq \kappa$. We shall show that $W=\bigcup\left\{U_{s} \times V_{s}: s \in S^{*}\right\}$.

Suppose that $(x, y) \in W$. Then $(x, y) \in U_{s} \times V_{s}$ for some $s \in S$. Hence, there exists $t \in T$ such that $y \in E_{t}$ and such that $\left|E_{t} \backslash V_{s}\right| \leq \kappa$. Because $s \in S_{t}$, there exists $s_{1} \in S_{t}^{*}$ such that $x \in U_{s_{1}}$. If $y \in G_{t}$, then $(x, y) \in U_{s_{1}} \times V_{s_{1}}$. On the other hand, if $y \notin G_{t}$ (in which case $y \in H$ ), then there exists $s_{2} \in S^{*}(y)$ 
such that $x \in U_{s_{2}}$, so that $(x, y) \in U_{s_{2}} \times V_{s_{2}}$. In either case, there exists $s \in S^{*}$ such that $(x, y) \in U_{s} \times V_{s}$. Hence, $X \times Y^{2}$ is hereditarily $\kappa$-Lindelöf.

2.4. Corollary. If spaces $X$ and $Y$ are such that $h l(X) \leq \kappa$ and $n w(Y) \leq \kappa$, then $h l(X \times Y) \leq \kappa$.

2.5. Corollary. Suppose that $\left\{X_{s}: s \in S\right\}$ is a collection of topological spaces such that

$$
\begin{gathered}
|S| \leq \kappa, \\
h l\left(X_{s_{0}}\right) \leq \kappa \quad \text { for some } s_{0} \in S,
\end{gathered}
$$

and

$$
p n\left(X_{s}\right) \leq \kappa \quad \text { for all } s \in S \backslash\left\{s_{0}\right\} .
$$

Then the Cartesian product $\prod\left\{X_{s}: s \in S\right\}$ is hereditarily $\kappa$-Lindelöf.

Proof. Using Theorem 1.5 and Corollary 1.9, we can inductively prove that if $S^{*}$ is a finite subset of $S$, then the space $\prod\left\{X_{s}: s \in S^{*}\right\}$ is hereditarily $\kappa$-Lindelöf. From Theorem 3 of [7] we immediately obtain the proposition.

2.6. Remarks. Applying a lemma of Juhász (cf. [7, Lemma 2, p. 176]), P. Zenor proved in [7, pp. 179-180] that the space $X_{0}$ considered earlier in Example 1.13 is such that $X_{0}^{\omega}$ is hereditarily Lindelöf. Let us observe that this property of $X_{0}$ is an immediate consequence of the preceding corollary. In like manner, the subspace $X_{2}$ in Example 1.14 also is Hausdorff, nonseparable, and such that $X_{2}^{\omega}$ is hereditarily Lindelöf.

\section{REFERENCES}

1. R. Engelking, General topology, PWN-Polish Scientific Publishers, Warsaw, 1977.

2. D. H. Fremlin, Consequences of Martin's axiom, Cambridge University Press, Cambridge, 1984.

3. A. Hajnal and I. Juhász, On hereditarily $\alpha$-Lindelöf and hereditarily $\alpha$-separable spaces, Ann. Univ. Sci. Budapest. Eötvös Sect. Math. 9 (1968), 115-124.

4. R. Hodel, Cardinal functions I, Handbook of Set-theoretic Topology (K. Kunen and J. E. Vaughan, eds.), North-Holland, Amsterdam, 1984, pp. 1-61.

5. I. Juhász, K. Kunen, and M. E. Rudin, Two more hereditarily separable non-Lindelöf spaces, Canad. J. Math. 28 (1976), 998-1005.

6. A. J. Ostaszewski, On countably compact, perfectly normal spaces, J. London Math. Soc. (2), 14 (1976), 505-516.

7. P. Zenor, Hereditary $m$-separability and the hereditary $m$-Lindelöf property in product spaces and function spaces, Fund. Math. 106 (1980), 175-180.

Current address (R. A. Johnson): Department of Mathematics, Washington State University, Pullman, Washington 99164-2930

Current address (E. Wajch and W. Wilczyński): Institute of Mathematics, University of Lodż, 90-238 Lodż, ul. Banacha 22, Poland 